\title{
PATTERN FORMATION IN THE NONLOCAL BISTABLE EQUATION
}

\author{
ADAM J. J. CHMAJ* AND XIAOFENG REN ${ }^{\dagger}$
}

\begin{abstract}
We study the nonlocal bistable equation that arises from a variational problem for binary materials with nonlocal constitutive relations. Using the singular perturbation $\Gamma$-convergence approach we prove the existence of 1-, 2-, and 3-layer local minimizers in one dimension for "mexican hat" nonlocalities. For some other nonlocalities we show the non-existence of non-constant stable solutions. We also present some numerical simulations of the equation in two dimensions.
\end{abstract}

1. Introduction. The theory of continuum mechanics postulates a set of constitutive relations when a particular material is studied. These relations give the inter-dependences among various phase fields that describe the material. They turn the mass, energy, momentum conservation laws to well posed evolution equations.

In the classical continuum mechanics theory, the constitutive relations are assumed to be local. One phase field variable at a place and a time depends on the other variables and/or their derivatives at the same place and the same time. Examples of such relations are pointwise linear (or nonlinear) strain-stress relations of elastic solids and pressure-density-temperature relations of the ideal, Abel and van der Walls gases.

This classical theory is however inadequate when more complex materials, such as martensites and di-block copolymers, are studied. These materials often have fine microstructures. The study of such materials require that we extend the classical theory to let nonlocal expressions enter their constitutive relations. In an earlier paper [7], we considered a single nonlocal constitutive relation, which can serve as a prototype for more sophisticated models. There we had two phase fields, the energy density of the continuum denoted by $e$ and a general phase field $u$.

The general phase field $u$ characterizes the state of the material. For twocomponent composite materials like copolymers, $u$ is often the relative concentration of one of the two components. The total energy of the material is defined to be

$$
I=\int_{\Omega} e(x) d x
$$

where $\Omega$ is the domain of the Euler or Lagrange coordinate $x$.

In order to see the constitutive relation between $e$ and $u$, we first recall the expression for the total energy in terms of the phase field $u$ from [7]

$$
I(u)=\frac{1}{4} \int_{\Omega} \int_{\Omega} J(x, y)(u(x)-u(y))^{2} d x d y+\int_{\Omega} W(u(x)) d x .
$$

We refer to $[4,5]$ for a derivation of this model by a statistical mechanics argument. The process is also summarized in [7].

We then rewrite (1.2) in an alternative form

$$
I(u)=\int_{\Omega}\left[-\frac{1}{2} J[u] u+\frac{j}{2} u^{2}+W(u)\right] d x
$$

${ }^{*}$ Department of Mathematics, Heriot-Watt University, Riccarton, Edinburgh EH14 4AS, Scotland, UK. Supported by a Marie Curie Fellowship of the European Community IHP programme under contract number HPMFCT-2000-00465 and in part by NSF grant DMS-0096182.

†Department of Mathematics and Statistics, Utah State University, Logan, UT 84322, USA. Supported in part by NSF grant DMS-9703727. 
where

$$
J[u](x)=\int_{\Omega} J(x, y) u(y) d y, \quad j(x)=\int_{\Omega} J(x, y) d y .
$$

Mathematically $J$ behaves as a kernel and $J[\cdot]$ is an integral operator. Comparing (1.1) and (1.3) we find the nonlocal constitutive relation

$$
e=-\frac{1}{2} J[u] u+\frac{j}{2} u^{2}+W(u)
$$

We now treat (1.2) or (1.3) as a variational problem. Stable configurations of the material are to be found as local minimizers of $I$. They satisfy the Euler-Lagrange equation

$$
-J[u]+j u+f(u)=0,
$$

where $f=W^{\prime}$. The dynamic counterpart of this model studied in this paper will simply be the negative $L^{2}$ gradient flow of $I$

$$
u_{t}=J[u]-j u-f(u)
$$

At this point we make a comparison to a local theory of Allen-Cahn [2] where the total energy is set to be

$$
\int_{\Omega}\left[\frac{1}{2}|\nabla u(x)|^{2}+W(u(x))\right] d x
$$

and whose corresponding negative $L^{2}$ gradient flow is

$$
u_{t}=\Delta u-f(u) \text { in } \Omega, \frac{\partial u}{\partial \nu}=0 \text { on } \partial \Omega .
$$

There is some resemblance between our nonlocal model (1.2)-(1.7) and the local AllenCahn model (1.8)-(1.9). At a formal level one can see (1.8) as a limiting case of $I$ as a non-negative, translationally invariant $J$ shrinks to a delta function (see e.g. [7]). We will often point out similarities and differences between these two models in this paper.

The choice of $J$ and $W$ in $I$ dictate the behaviors of the local minimizers of $I$ and the solutions of (1.7). Several choices of $J$ are possible. Regarding the sign of $J$, the simplest case is that $J(x, y) \geq 0$ for all $x, y \in \Omega$. As was derived in [4], it also makes sense to study the $J$ 's that change sign on $\Omega \times \Omega$. The latter case is used e.g., when we apply this theory to di-block copolymers (see [12]).

$J$ is always symmetric, in the sense $J(x, y)=J(y, x)$ for all $x, y \in \Omega$. However there is first the case where $J$ is translationally invariant, i.e. $J(x, y)=J(x-y)$, which corresponds to the situation where the environment outside the boundary has no effect on the behavior of the energy density $e$, and the second case where $J$ is not translationally invariant, which corresponds to the situation where the environment outside the boundary interacts with the material and brings some contribution to $e$.

The local part of the energy, $W$, is non-convex in this paper. One particular case of interest is when $W$ has two global minima at, say -1 and 1 , such that $W(-1)=W(1)$. In this paper we will only consider small perturbations of this special case.

The study of equation (1.7) on the whole line was initiated by Bates, Fife, Ren and Wang in [3]. Assuming $J \geq 0$, traveling waves were constructed, and it was discovered 
that stationary waves can be discontinuous. Later in [6], we found unstable stationary solutions of homoclinic type for $J \geq 0$ and $J(x)$ decreasing for $x \in(0, \infty)$. In [4], Bates and Chmaj studied the case in which the effect of $J$ is much weaker than that of $W$ and found stable stationary solutions which are "pinned" to the zeros of $f$ and discontinuous across arbitrarily prescribed interfaces. They also studied a discrete analogy of $I$ in [5].

This paper continues the work started in [7]. We assume that $J$ can be roughly separated into two scales. When $|x-y|$ is at a smaller scale, say of magnitude $\epsilon, J$ is positive and of order $1 / \epsilon^{d}$, where $d$ is the dimension of $\Omega$. When $|x-y|$ is at a scale larger than $\epsilon, J$ is of order $\epsilon$. This separation of scales enables us to take up a singular perturbation approach to the variational problem $I$. We base our work on the theory of $\Gamma$-convergence of De Giorgi [9]. This type of convergence for $I$ was established in the work of Alberti and Bellettini [1]. Physically, the behavior of $J$ for small $|x-y|$ penalizes oscillations for any configuration $u$. But the unspecified behavior of $J$ for larger $|x-y|$ leads to various interesting patterns of $u$. Local minima constructed this way all take values near the two minima of $W$, and often oscillate between these two values, therefore having transitional regions. In one dimension such regions are characterized by a finite set of points. In a neighborhood whose width is of order $\epsilon$, around such a point a local minimum of $I$ sees its value change from one minimum value to another minimum value of $W$. In two dimensions our numerical computation often finds the transitional regions to be a set of curves whose width is of order $\epsilon$. Again a local minimum of $I$ changes its value from one minimum value to another minimum value of $W$ as it crosses such a curve.

For more specific examples of materials modeled by functionals of type (1.2) we refer to the papers of Ren and Truskinovsky [11] that studied martensites, and of Ren and Wei [12] that studied di-block copolymers.

This paper is organized as follows. In Section 2 we summarize the main results obtained in [7] and indicate where we will go from there. In Section 3 we rigorously construct stable solutions of (1.6) in one dimension. Then in Section 4 we present some numerical simulations of (1.7) in two dimensions.

2. A review of the $\Gamma$-convergence approach. We first precisely formulate the two scales in $J$. Assume that

$$
J(x, y)=J_{\epsilon}^{s}(|x-y|)-\epsilon J^{l}(x, y)=\frac{1}{\epsilon^{d}} J^{s}\left(\frac{|x-y|}{\epsilon}\right)-\epsilon J^{l}(x, y),
$$

where $\epsilon>0$ and $J_{\epsilon}^{s}(|x-y|)=\frac{1}{\epsilon^{d}} J^{s}\left(\frac{|x-y|}{\epsilon}\right)$ describes the behavior of $J$ at $|x-y|=O(\epsilon)$ and $\epsilon J^{l}(x, y)$ the behavior of $J$ at $|x-y|=O(1)$. Four conditions on $J^{s}$ and $J^{l}$ are imposed.

H1 The short range effect is translationally invariant, even and penalizes oscillation, i.e. $J^{s}(-r)=J^{s}(r) \geq 0, \int_{R^{d}} J^{s}(|x|) d x>0$

H2 On the growth of $J^{s}$ we assume $\int_{R} J^{s}(r) d r<\infty, \int_{R}|r| J^{s}(r) d r<\infty$.

H3 $J^{l}$ is symmetric, i.e. $J^{l}(x, y)=J^{l}(y, x)$. The function $j^{l}(x)=\int_{\Omega} J^{l}(x, y) d y$ is in $L^{\infty}(\Omega)$.

H4 The operator $J: u \rightarrow J[u]$ is compact from $L^{2}(\Omega)$ to itself. This condition is satisfied if, e.g., $J \in L^{2}(\Omega \times \Omega)$.

Note that at this point the sign of $J^{l}$ is not specified. The local term $f$ is continuous and allowed to have an expansion in $\epsilon, f=f_{0}+\epsilon f_{1}$. Let $W(s)=\int_{-1}^{s} f(r) d r$. In terms of $\epsilon, W=W_{0}+\epsilon W_{1}$, where $W_{0}(s)=\int_{-1}^{s} f_{0}(r) d r$ and $W_{1}(s)=\int_{-1}^{s} f_{1}(r) d r$. The conditions on $f$ are imposed on $W$. 
H5 $W_{0}$ is a double well function of equal depth. So $W_{0}(s) \geq 0$ for all $s \in R$, and only when $s= \pm 1, W_{0}(s)=0$, i.e. $\int_{-1}^{1} f_{0}(r) d r=0$.

H6 The growth rate of $W_{0}$ is at least quadratic, i.e., there exists $C>0$ such that $W_{0}(s) \geq C s^{2}-C$ for all $s \in R$.

H7 The growth rate of $W_{1}$ is at most quadratic, i.e., there exists $C>0$ such that $\left|W_{1}(s)\right| \leq C|s|^{2}+C$ for all $s \in R$.

H8 For small $\epsilon, \frac{j(x) s^{2}}{2}+W(s)$ is convex in $s$ for all $x$.

The conditions H1-8 will hold throughout this paper. The last condition, H8, may seem strange. It is actually quite important, for it provides the well-posedness of $I$. For example only under this condition the functional $I$ is weakly lower semicontinuous. It also guarantees that solutions of (1.6) are continuous.

In connection with the form of $J$ set in (2.1), we denote the functional $I$ defined in (1.2) by $I_{\epsilon}$ to stress the dependence on the perturbation parameter $\epsilon$.

Proposition 2.1. $I_{\epsilon}$ of (1.2) defines a functional: $L^{2}(\Omega) \rightarrow R$, with the following properties.

1. There exists a constant $c$ independent of small $\epsilon$ so that for every $u \in L^{2}(0,1)$, $I_{\epsilon}(u) \geq c \epsilon$.

2. There exists $C>0$, independent of small $\epsilon$, so that $\|u\|_{2} \leq C I_{\epsilon}(u)+C$.

3. For small $\epsilon, I_{\epsilon}$ is weakly lower semi-continuous on $L^{2}(\Omega)$.

4. $I_{\epsilon}$ has a global minimum if $\epsilon$ is small.

5. On any convex $X \subset L^{2}(\Omega)$, for small $\epsilon$ there exists $u_{X} \in \bar{X}$ such that $I_{\epsilon}\left(u_{X}\right)=\inf _{u \in X} I_{\epsilon}(u)$.

Proof. See [7], Proposition 2.1.

Under this setting, we can take a singular perturbation approach to the study of $I_{\epsilon}$. It turns out that the functional $\epsilon^{-1} I_{\epsilon}$ has a well defined singular limit $K+I^{l}$. This limit is defined on $L^{2}(\Omega)$, though only for $u \in B V(\Omega,\{-1,1\})\left(K+I^{l}\right)(u)$ is finite. Here $B V(\Omega,\{-1,1\})$ is the space of functions of finite variation with the range $\{-1,1\}$. The formal definition of $K$ is

$$
K(u)=\left\{\begin{array}{ll}
c_{0} \frac{\|D u\|(0,1)}{2} & \text { if } u \in B V(\Omega,\{-1,1\}) \\
\infty & \text { otherwise }
\end{array} .\right.
$$

$I^{l}$ is the $\epsilon$ perturbation part of of $I_{\epsilon}$. For $u \in L^{2}(\Omega)$,

$$
\begin{aligned}
I^{l}(u) & =-\frac{1}{4} \int_{0}^{1} \int_{0}^{1} J^{l}(x, y)(u(x)-u(y))^{2} d x d y+\int_{0}^{1} W_{1}(u) d x \\
& =\int_{\Omega}\left[\frac{1}{2} J^{l}[u] u-\frac{j^{l} u^{2}}{2}+W_{1}(u)\right] d x .
\end{aligned}
$$

Here $\|D u\|$ is the absolute value of the distributional derivative $D u$ of $u$, regarded as a finite nonnegative measure on $\Omega$. $\|D u\|(\Omega)$ is the size of $\Omega$ under this measure. Intuitively this is twice the combined area of the hyper-surfaces in $\Omega$ that divide the region $\{x \in \Omega: u(x)=-1\}$ from the region $\{x \in \Omega: u(x)=1\}$.

The constant $c_{0}$ depends on the solution of

$$
-J^{s} * U+\left(\int_{R} J^{s}(r) d r\right) U+f_{0}(U)=0 \text { in } R, U( \pm \infty)= \pm 1
$$


The existence and uniqueness, up to translation, of such a solution is shown in [3]. Then $c_{0}$ is a positive number equal to

$$
\frac{1}{4} \int_{R} \int_{R} J^{s}(y, r)(U(y)-U(r))^{2} d y d r+\int_{R} W_{0}(U(r)) d r
$$

The next proposition describes the convergence of $\epsilon^{-1} I_{\epsilon}$ to $K+I^{l}$.

Proposition 2.2. $\epsilon^{-1} I_{\epsilon} \Gamma$-converges to $K+I^{l}$ as $\epsilon \rightarrow 0$ in the following sense.

1. For every $\left\{u_{\epsilon}\right\} \subset L^{2}(\Omega)$ with $\lim _{\epsilon \rightarrow 0} u_{\epsilon}=u$ in $L^{2}(\Omega)$,

$$
\liminf _{\epsilon \rightarrow 0} \epsilon^{-1} I_{\epsilon}\left(u_{\epsilon}\right) \geq\left(K+I^{l}\right)(u)
$$

2. For every $u \in L^{2}(\Omega) \cap B V((\Omega),\{-1,1\})$, there exists a family $\left\{u_{\epsilon}\right\} \subset L^{2}(\Omega)$ such that $\lim _{\epsilon \rightarrow 0} u_{\epsilon}=u$ in $L^{2}(\Omega)$, and

$$
\limsup _{\epsilon \rightarrow 0} \epsilon^{-1} I_{\epsilon}\left(u_{\epsilon}\right) \leq\left(K+I^{l}\right)(u)
$$

Proof. See [7], Proposition 2.2. and [1].

The most important property we use from the $\Gamma$-convergence theory is that around every strict local minimum of $K+I^{l}$ there exists a local minimum of $I_{\epsilon}$. Let us denote an open ball in $L^{2}(\Omega)$ of radius $\delta$ around a function $u_{0}$ by $B_{\delta}\left(u_{0}\right)$.

Proposition 2.3. Let $\delta>0$ and $u_{0} \in L^{2}(\Omega)$ be such that $\left(K+I^{l}\right)\left(u_{0}\right)<$ $\left(K+I^{l}\right)(u)$ for all $u \in B_{\delta}\left(u_{0}\right)$ with $u \neq u_{0}$. Then for small $\epsilon$ there exists $u_{\epsilon} \in B_{\delta / 2}\left(u_{0}\right)$ with $I_{\epsilon}\left(u_{\epsilon}\right) \leq I_{\epsilon}(u)$ for all $u \in B_{\delta / 2}\left(u_{0}\right)$. In addition $\lim _{\epsilon \rightarrow 0}\left\|u_{\epsilon}-u_{0}\right\|_{2}=0$.

Proof. See [7], Proposition 2.4 and [13]. The proof relies on Proposition 2.2 above and a coercivity property of $I_{\epsilon}$, holding for $J^{s} \geq 0$ [1]: If $\epsilon_{n}$ is a sequence of positive numbers converging to $0,\left\{u_{n}\right\}$ a sequence in $L^{2}(0,1)$ and $\epsilon_{n}^{-1} I_{\epsilon_{n}}\left(u_{n}\right)$ are bounded above in $n$, then $\left\{u_{n}\right\}$ is relatively compact in $L^{2}(0,1)$.

In [7] after establishing the general $\Gamma$-convergence theory for $I_{\epsilon}$, we turned our attention to the case $\Omega=(0,1)$. Then every member $u$ in $B V((0,1),\{-1,1\})$ can be identified by a set of points where $u$ jumps between -1 and 1 . More precisely, let

$$
A_{N}=\left\{u \in B V((0,1),\{-1,1\}): \frac{\|D u\|(0,1)}{2}=N\right\}
$$

the set of functions with $N$ jumps. This set can be further divided into $A_{N}^{-1}$ and $A_{N}^{1}$.

$$
\begin{aligned}
A_{N}^{-1} & =\left\{u \in A_{N}: u(x)=-1, \text { if } x \text { is between } 0\right. \\
& \text { and the first jump point of } u\} \\
\cong & \left\{\left(\xi_{1}, \xi_{2}, \ldots, \xi_{N}\right): 0<\xi_{1}<\xi_{2}<\ldots<\xi_{N}<1\right\} \\
A_{N}^{1}= & \left\{u \in A_{N}: u(x)=1, \text { if } x \text { is between } 0\right. \\
& \text { and the first jump point of } u\} \\
\cong & \left\{\left(\xi_{1}, \xi_{2}, \ldots, \xi_{N}\right): 0<\xi_{1}<\xi_{2}<\ldots<\xi_{N}<1\right\}
\end{aligned}
$$

Note $A_{0}^{-1}=\{-1\}$ and $A_{0}^{1}=\{1\}$. Now we have a mutually disjoint decomposition

$$
B V((0,1),\{-1,1\})=\cup_{N=0}^{\infty}\left(A_{N}^{-1} \cup A_{N}^{1}\right)
$$


The next proposition shows that every local minimum of $I^{l}$ restricted on one of the $A_{N}^{ \pm 1}$ 's is also a local minimum of $K+I^{l}$ on $L^{2}(0,1)$. Therefore finding local minima of $K+I^{l}$ becomes a minimization problem on a union of finite dimensional spaces $A_{N}^{ \pm 1}$.

Proposition 2.4. If $\chi \in A_{N}^{ \pm 1}$ is a strict local minimum of $I^{l}$ restricted on $A_{N}^{ \pm 1}$, then $u$ is a strict local minimum of $K+I^{l}$ defined on $L^{2}(0,1)$.

Proof. We only consider the case $\chi \in A_{N}^{-1}$. Take a function $u \in B_{\delta}(\chi) \subset L^{2}(0,1)$, $u \neq \chi, \delta$ to be specified later. If $u \in A_{N}^{-1}$, then $\left(K+I^{l}\right)(u)>\left(K+I^{l}\right)(\chi)$ by the assumption of this proposition. If $u \in L^{2}(0,1) \backslash B V((0,1),\{-1,1\})$, then $\infty=\left(K+I^{l}\right)(u)>\left(K+I^{l}\right)(\chi)$. We now consider the remaining case $u \in$ $B V((0,1),\{-1,1\}) \backslash A_{N}^{-1}$. In this case $\frac{\|D u\|}{2}$, the number of jumps $u$ has, is either $\geq N+1$ or $\leq N-1$. The sub-case $\frac{\|D u\|}{2} \leq N-1$ does not happen by a property of BV-functions (see [10] Theorem 1, p. 172): If $u_{n} \rightarrow \chi$ in $L^{1}$ then $\liminf _{n \rightarrow \infty}\left\|D u_{n}\right\|(0,1) \geq\|D \chi\|(0,1)=2 N$. So if we choose $\delta$ small, $\frac{\|D u\|(0,1)}{2}$ can not be less than or equal to $N-1$. In the sub-case $\frac{\|D u\|}{2} \geq N+1$,

$$
\begin{aligned}
\left(K+I^{l}\right)(u) & \geq c_{0}(N+1)+I^{l}(u) \\
& =\left(K+I^{l}\right)(\chi)+I^{l}(u)-I^{l}(\chi)+c_{0} \\
& >\left(K+I^{l}\right)(\chi)
\end{aligned}
$$

if $\delta$ is chosen small enough, by the continuity of $I^{l}$.

This proposition is used in combination with Proposition 2.3 to find local minima of $I_{\epsilon}$ near members of $A_{N}^{ \pm 1}$. Since a member of $A_{N}^{ \pm 1}$ jumps between -1 and $1 N$ times, any local minimum of $I_{\epsilon}$ found near such a member must have $N$ layers located around the jump points of the member of $A_{N}^{ \pm 1}$

In $A_{0}^{ \pm 1}$ the lone element element \pm 1 is a local minimum. To find local minima in other $A_{N}^{ \pm 1}$, we identify every $u \in A_{N}^{ \pm 1}, N \geq 1$ by its jump points, $\xi_{1}, \xi_{2}, \ldots, \xi_{N}$. Then $\left(K+I^{l}\right)(u)=c_{0} N+I^{l}(u)$ can be expressed as a function of $\xi_{i}$. The partial derivatives of this function can be calculated as in [7] to obtain

$$
\frac{\partial I^{l}(u)}{\partial \xi_{i}}=\left\{\begin{aligned}
(-1)^{i} 2 J^{l}[u]\left(\xi_{i}\right)+(-1)^{i} \int_{-1}^{1} f_{1}(r) d r, & u \in A_{N}^{-1} \\
-(-1)^{i} 2 J^{l}[u]\left(\xi_{i}\right)-(-1)^{i} \int_{-1}^{1} f_{1}(r) d r, & u \in A_{N}^{1}
\end{aligned}\right.
$$

However solving $\partial I^{l}(u) / \partial \xi_{i}=0$ for general $J^{l}$ is by no means easy. In [7] we only took a very special $J^{l}$, which was used e.g., in the work of Ren and Truskinovsky [11]. We assumed

$$
J^{l}(x, y)=\frac{1}{\gamma\left(e^{\frac{1}{\gamma}}-e^{-\frac{1}{\gamma}}\right)}\left[\cosh \left(\frac{x+y-1}{\gamma}\right)+\cosh \left(\frac{|x-y|-1}{\gamma}\right)\right], \gamma>0
$$

which is Green's function of the linear differential equation $-\gamma^{2} v^{\prime \prime}+v=u, v^{\prime}(0)=$ $v^{\prime}(1)=0$. We also needed the condition

$$
-2<\int_{-1}^{1} f_{1}(r) d r<2
$$

Then we proved (Theorem 2.7 in [7])

THEOREM 2.5. Let $J^{l}$ be defined by (2.8) and $f_{1}$ satisfy (2.9). For each positive integer $\nu$ we can find $\delta>0$ and $\chi_{N}^{ \pm 1} \in A_{N}^{ \pm 1}, N=1,2, \ldots, \nu$, such that 
1. $\left\{B_{\delta}\left(\chi_{N}^{-1}\right), B_{\delta}\left(\chi_{N}^{1}\right): N=1,2, \ldots, \nu\right\}$ is a family of $2 \nu$ mutually disjoint open balls in $L^{2}(0,1)$;

2. for small $\epsilon$, there exist a local minimum $u_{N, \epsilon}^{-1}$ of $I_{\epsilon}$ in $B_{\delta}\left(\chi_{N}^{-1}\right)$ and a local minimum $u_{N, \epsilon}^{1}$ in $B_{\delta}\left(\chi_{N}^{1}\right)$, for $N=0,1, \ldots \nu$, satisfying $\lim _{\epsilon \rightarrow 0}\left\|u_{N, \epsilon}^{-1}-\chi_{N}^{-1}\right\|_{2}=0$ and $\lim _{\epsilon \rightarrow 0}\left\|u_{N, \epsilon}^{1}-\chi_{N}^{1}\right\|_{2}=0$.

Here $\chi_{N}^{ \pm 1}$ in $A_{N}^{ \pm 1}$ are the local minima of $I^{l}$. Because of the specialty of $j^{l}$, it was proved in [7] Section 2, that the $N$ jump points $\xi_{i}$ of $\chi_{N}^{ \pm 1}$ are regularly distributed on $(0,1)$, in the serse that

$$
2 \xi_{1}=\xi_{3}-\xi_{2}=\xi_{5}-\xi_{4}=\ldots, \quad \xi_{2}-\xi_{1}=\xi_{4}-\xi_{3}=\xi_{6}-\xi_{5}=\ldots
$$

The $\xi_{1}$ of $\chi_{N}^{-1}$ is the unique solution of

$$
\left[1-\frac{1}{2} \int_{-1}^{1} f_{1}(r) d r\right] \tanh \left(\frac{\xi_{1}}{\gamma}\right)=\left[1+\frac{1}{2} \int_{-1}^{1} f_{1}(r) d r\right] \tanh \left(\frac{1}{\gamma N}-\frac{\xi_{1}}{\gamma}\right)
$$

and the $\xi_{1}$ of $\chi_{N}^{1}$ is the unique solution of

$$
\left[1+\frac{1}{2} \int_{-1}^{1} f_{1}(r) d r\right] \tanh \left(\frac{\xi_{1}}{\gamma}\right)=\left[1-\frac{1}{2} \int_{-1}^{1} f_{1}(r) d r\right] \tanh \left(\frac{1}{\gamma N}-\frac{\xi_{1}}{\gamma}\right)
$$

In the next section we consider $J^{l}$ more general than (2.8). We can not expect the jump points of the local minima of $I^{l}$ to be regularly distributed as in (2.10). The function $J^{l}$ defined in $(2.8)$ is positive on $(0,1) \times(0,1)$. In the next section we consider both the case where $J^{l} \geq 0$ and the case where $J^{l}$ changes sign.

3. Stationary solutions on $(0,1)$. In this section we consider a class of more general $J^{l}$ 's. First is the rather trivial case of the existence of almost constant local minima of $I_{\epsilon}$.

THEOREM 3.1. For sufficiently small $\epsilon, I_{\epsilon}$ has a local minimum close to -1 and a local minimum close to 1 .

Proof. In $A_{0}^{ \pm 1}$ the lone element $u= \pm 1$ is trivially a local minimum. The theorem then follows from Propositions 2.4 and 2.3.

However local minima of $I_{\epsilon}$ with layers, i.e., local minima close to some members of $A_{N}^{ \pm 1}, N \geq 1$, are much harder to find. To make the problem more tractable, we assume that $J^{l}$ is translationally invariant, in the sense $J^{l}(x, y)=J^{l}(x-y)=J^{l}(y-x)$. This excludes any influence to $u$ from the outside of the region $\Omega$. We also need a counterpart of (2.9), which here is

$$
\left|\int_{-1}^{1} f_{1}(r) d r\right|<2 j^{l}(0)=2 \int_{0}^{1} J^{l}(x) d x .
$$

Using the special $J^{l}$ in (2.7), we were able to solve $\partial I^{l}(u) / \partial \xi_{i}=0$ in $A_{N}^{ \pm 1}$ to obtain a unique solution $\chi_{N}^{ \pm 1}$. Then this uniqueness was used to show stability [7]. For general $J^{l}(x-y)$, we expect a similar uniqueness result to be hard to obtain. Therefore, after solving $\partial I^{l}(u) / \partial \xi_{i}=0$ we find it necessary to analyze the second derivative (the Hessian matrix) of $I^{l}$ with respect to $\xi_{i}$. Following (2.7) we find

$$
\frac{1}{2} \frac{\partial^{2} I^{l}(u)}{\partial \xi_{i} \partial \xi_{j}}=\left\{\begin{aligned}
(-1)^{i}\left[-J^{l}\left(\xi_{i}\right)+\sum_{k \neq i}(-1)^{k+1} 2 J^{l}\left(\xi_{k}-\xi_{i}\right)+(-1)^{N} J^{l}\left(1-\xi_{i}\right)\right] & \text { if } i=j \\
2(-1)^{i+j} J^{l}\left(\xi_{i}-\xi_{j}\right), & \text { if } i \neq j
\end{aligned}\right.
$$


We first consider the case that $J^{l} \geq 0$. Therefore the entire kernel $J=J_{\epsilon}^{s}-\epsilon J^{l}$ changes sign once for small $\epsilon$. If $J^{l}$ is decreasing, $J$ takes the form which is known in the literature (e.g. [14]) as the "mexican hat".

THEOREM 3.2. Let $J^{l}$ be continuous, with $J^{l} \geq 0$ on $(-1,1)$, and $J^{l}>0$ on $[-1 / 2,1 / 2]$. If (3.1) holds, then for small $\epsilon I_{\epsilon}$ has a local minimum near a unique member of $A_{1}^{-1}$ and a local minimum near a unique member of $A_{1}^{1}$.

Proof. Based on Propositions 2.4 and 2.3, we need only to find zeros of (2.7) that make (3.2) positive definite. In $A_{1}^{-1} \partial I^{l}(u) / \partial \xi_{1}=0$ becomes

$$
\int_{0}^{\xi_{1}} J^{l}(y) d y=\int_{0}^{1-\xi_{1}} J^{l}(y) d y+\frac{1}{2} \int_{-1}^{1} f_{1}(r) d r .
$$

Denote the left side of (3.3) by $l_{1}\left(\xi_{1}\right)$, the right side by $r_{1}\left(\xi_{1}\right)$. Note that by (3.1)

$$
\begin{gathered}
0=l_{1}(0)<r_{1}(0)=j^{l}(0)+\frac{1}{2} \int_{-1}^{1} f_{1}(r) d r \\
j^{l}(0)=l_{1}(1)>r_{1}(1)=\frac{1}{2} \int_{-1}^{1} f_{1}(r) d r .
\end{gathered}
$$

$l_{1}$ and $r_{1}$ are continuous functions, with $l_{1}$ non-decreasing and $r_{1}$ non-increasing. Moreover on interval $[0,1 / 2] l_{1}$ is strictly increasing and on $[1 / 2,1] r_{1}$ is strictly decreasing. There is a unique $\xi_{1}$ such that $l_{1}\left(\xi_{1}\right)=r_{1}\left(\xi_{1}\right)$. The Hessian (3.2) at $\xi_{1}$ is $J^{l}\left(\xi_{1}\right)+J^{l}\left(1-\xi_{1}\right)>0$ since at least one of $J^{l}\left(\xi_{1}\right)$ and $J^{l}\left(1-\xi_{1}\right)$ is positive. Therefore $\xi_{1}$ is a local minimum. Thus there is a critical point corresponding to $\xi_{1}$. Note that if $\int_{-1}^{1} f_{1}(r) d r=0$, then $\xi_{1}=\frac{1}{2}$. The same argument applies to the case $A_{1}^{1}$.

THEOREM 3.3. In addition to the assumptions on $J^{l}$ in Theorem 3.2 if we assume that $J^{l}(x)$ is decreasing on $(0,1)$ with $J_{x}^{l}(1 / 2)<0$, then for small $\epsilon I_{\epsilon}$ has a local minimum near a unique member of $A_{2}^{-1}$ and a local minimum near a unique member of $A_{2}^{1}$.

Proof. First we establish uniqueness, i.e., we show that any solution to the equations $\partial I^{l}(u) / \partial \xi_{i}=0, i=1,2$, must be such that $\xi_{1}=1-\xi_{2}$. Note that these equations can be written as

$$
\begin{aligned}
& -\int_{0}^{\xi_{1}} J^{l}(y) d y+\int_{0}^{\xi_{2}-\xi_{1}} J^{l}(y) d y-\int_{\xi_{2}-\xi_{1}}^{1-\xi_{1}} J^{l}(y) d y=-\frac{1}{2} \int_{-1}^{1} f_{1}(r) d r \\
& -\int_{0}^{1-\xi_{2}} J^{l}(y) d y+\int_{0}^{\xi_{2}-\xi_{1}} J^{l}(y) d y-\int_{\xi_{2}-\xi_{1}}^{\xi_{2}} J^{l}(y) d y=-\frac{1}{2} \int_{-1}^{1} f_{1}(r) d r
\end{aligned}
$$

Without loss of generality assume that $\xi_{1}<1 / 2, \xi_{2}>1 / 2$ and $1-\xi_{2}>\xi_{1}$. Subtracting the first equation from the second, we get

$$
\int_{0}^{1-\xi_{2}-\xi_{1}} J^{l}(y) d y=\int_{\xi_{2}}^{1-\xi_{1}} J^{l}(y) d y .
$$


Since we assumed that $J_{x}^{l}(1 / 2)<0$, we obtain a contradiction. Thus $\xi_{1}=1-\xi_{2}$, and the above two equations reduce to one

$$
-\int_{0}^{\xi_{1}} J^{l}(y) d y+\int_{0}^{1-2 \xi_{1}} J^{l}(y) d y-\int_{1-2 \xi_{1}}^{1-\xi_{1}} J^{l}(y) d y=-\frac{1}{2} \int_{-1}^{1} f_{1}(r) d r .
$$

Denote the left side of (3.4) by $l_{2}\left(\xi_{1}\right)$. Then $l_{2}(0)=j^{l}(0)$,

$$
l_{2}\left(\frac{1}{2}\right)=-2 \int_{0}^{1 / 2} J^{l}(y) d y<-j^{l}(0)
$$

since $J^{l}$ being decreasing on $(0,1)$ with $J_{x}^{l}(1 / 2)<0$ implies that $\int_{0}^{1 / 2} J^{l}>\int_{1 / 2}^{1} J^{l}$. Thus by (3.1) (3.4) has a solution $\xi_{1} \in(0,1 / 2)$. The Hessian (3.2) for the critical point $\chi_{2}^{-1}$ is

$$
\left[\begin{array}{cc}
J^{l}\left(\xi_{1}\right)+2 J^{l}\left(\xi_{2}-\xi_{1}\right)-J^{l}\left(1-\xi_{1}\right) & -2 J^{l}\left(\xi_{2}-\xi_{1}\right) \\
-2 J^{l}\left(\xi_{2}-\xi_{1}\right) & J^{l}\left(1-\xi_{2}\right)+2 J^{l}\left(\xi_{2}-\xi_{1}\right)-J^{l}\left(\xi_{2}\right)
\end{array}\right] .
$$

It is easily verified that $J_{x}^{l}(1 / 2)<0$ implies that (3.5) is positive definite, thus $\chi_{2}^{-1}$ is a local minimum.

THEOREM 3.4. In addition to the assumptions in Theorem 3.3 we assume that $\int_{-1}^{1} f_{1}(r) d r=0$ and $J^{l}(1 / 4)>2 J^{l}(1 / 2)$. Then for small $\epsilon I_{\epsilon}$ has a local minimum near a member of $A_{3}^{-1}$, and a local minimum near a member of $A_{3}^{1}$.

Proof. We consider the case $A_{3}^{-1}$. Look for a zero of (2.7) in the form $\xi_{1} \in$ $(0,1 / 2), \xi_{2}=1 / 2, \xi_{3}=1-\xi_{1}$. The three equations $\partial I^{l}(u) / \partial \xi_{i}=0, i=1,2,3$, reduce to one

$$
-\int_{0}^{\xi_{1}} J^{l}(y) d y+\int_{0}^{\frac{1}{2}-\xi_{1}} J^{l}(y) d y-\int_{\frac{1}{2}-\xi_{1}}^{1-2 \xi_{1}} J^{l}(y) d y+\int_{1-2 \xi_{1}}^{1-\xi_{1}} J^{l}(y) d y=0
$$

Denote the left side of (3.6) by $l_{3}\left(\xi_{1}\right)$. Then $l_{3}(0)=\int_{0}^{\frac{1}{2}} J^{l}-\int_{\frac{1}{2}}^{1} J^{l}>0$ and $l_{3}\left(\frac{1}{4}\right)=$ $-\int_{\frac{1}{4}}^{\frac{1}{2}} J^{l}+\int_{\frac{1}{2}}^{\frac{3}{4}} J^{l}<0$. Thus there exists a solution $\xi_{1}<\frac{1}{4}$. Note that the assumption $J^{l}(1 / 4)>2 J^{l}(1 / 2)$ is not needed for this existence.

The Hessian (3.2) at this solution is

$$
\left[\begin{array}{rcr}
a & -b & c \\
-b & -2 J^{l}\left(\xi_{2}\right)+2 b & -b \\
c & -b & a
\end{array}\right]
$$

where $a=J^{l}\left(\xi_{1}\right)+J^{l}\left(\xi_{3}\right)+b-c, b=2 J^{l}\left(\xi_{2}-\xi_{1}\right), c=2 J^{l}\left(\xi_{3}-\xi_{1}\right)$. To verify that (3.7) is positive definite, we show that the determinants of the three nested (at $a$ ) principal minors are positive. Since $b>c, a>0$. The second determinant is equal to $a\left[-2 J^{l}\left(\xi_{2}\right)+2 b\right]-b^{2}>a b-b^{2}$. Note that $a-b=J^{l}\left(\xi_{1}\right)+J^{l}\left(\xi_{3}\right)-2 J^{l}\left(\xi_{3}-\xi_{1}\right)$. Since $\xi_{1}<\frac{1}{4}$ and $J^{l}\left(\frac{1}{4}\right)>2 J^{l}\left(\frac{1}{2}\right)$, the second determinant is positive. The third is equal to

$$
\begin{aligned}
& (a-c)\left[\left(-2 J^{l}\left(\xi_{2}\right)+2 b\right)(a+c)-2 b^{2}\right] \\
& =(a-c)\left[\left(-2 J^{l}\left(\xi_{2}\right)+2 b\right)\left(J^{l}\left(\xi_{1}\right)+J^{l}\left(\xi_{3}\right)\right)-2 b J^{l}\left(\xi_{2}\right)\right] \\
& =(a-c) 2 J^{l}\left(\xi_{2}-\xi_{1}\right)\left[J^{l}\left(\xi_{1}\right)-2 J^{l}\left(\xi_{2}\right)+J^{l}\left(\xi_{3}\right)\right] .
\end{aligned}
$$


Since $J^{l}\left(\frac{1}{4}\right)>2 J^{l}\left(\frac{1}{2}\right), a-c$ and $J^{l}\left(\xi_{1}\right)-2 J^{l}\left(\xi_{2}\right)+J^{l}\left(\xi_{3}\right)$ are both positive, so is this determinant. Thus (3.7) is positive definite and $\chi_{3}^{-1}$ is a local minimum.

For $J^{l}(x)=b-m|x|, b \geq m$, it can be easily calculated that $\xi_{1}=1 / 6, \xi_{2}=1 / 2$, $\xi_{3}=5 / 6$ and that this solution is stable, even though the condition $J^{l}(1 / 4)>2 J^{l}(1 / 2)$ does not hold.

It is likely that for $J^{l}$ as in Theorem $3.2, A_{3}^{-1}$ has a unique solution, which is of the form $\xi_{1} \in(0,1 / 2), \xi_{2}=1 / 2, \xi_{3}=1-\xi_{1}$. If this is the case, then the following counterexample shows that not for all decreasing $J^{l}$ 's the three layer solution is stable, and one can in general expect minimizers with up to only two layers.

Let $J^{l}(x)=1-x^{6}$ (note that for this $J^{l}$ the condition $J^{l}(1 / 4)>2 J^{l}(1 / 2)$ does not hold). Then $\partial I^{l}(u) / \partial \xi_{i}, i=1,2,3$, is a system of three quadratic equations for $\xi_{1}, \xi_{2}, \xi_{3}$. It is easy to solve it numerically using e.g., Maple. We find the solution to be $\xi_{1}=.08530529970, \xi_{2}=.5, \xi_{3}=.9146947003$. Then we get $a-c=.7061520272$ and $J^{l}\left(\xi_{1}\right)-2 J^{l}\left(\xi_{2}\right)+J^{l}\left(\xi_{3}\right)=-.5544257772$, making the determinant of the Hessian (3.7) negative, thus the solution is unstable.

We next consider the situation where $J^{l}$ changes sign once on $(0,1)$. Since we are interested in cases where $J=J_{\epsilon}^{s}-\epsilon J^{l}$ is of mexican hat type, we focus our attention on the case where $-J^{l}$ also has a mexican hat shape. Note the difference from the previous case: if $J^{l}$ is decreasing on $(0,1)$, the zero of $J$ tends to 0 as $\epsilon \rightarrow 0$. If $-J^{l}$ has a mexican hat shape, the zero of $J$ tends to the zero of $J^{l}$ as $\epsilon \rightarrow 0$. In Theorem 3.7 below we show that $I$ does not admit local minimizers for $J(x)=b-m|x|$ with $b, m>0$ and $b \geq 3 m / 4$. This $J$ can change sign, but always its zero is $\geq 3 / 4$. This suggests that for $J$ changing sign the existence of local minimizers may depend on the location of the zero of $J$. This is indeed the case in what follows. For simplicity we study only solutions in $A_{1}^{-1}$.

THEOREM 3.5. Let $\int_{0}^{1} f_{1}(r) d r=0$. Suppose $J^{l}(x)<0$ for $0 \leq x<x_{0}$ and $J^{l}(x)>0$ for $x>x_{0}$. Then, the following holds:

If $x_{0}>1 / 2$ and $\int_{0}^{1} J^{l}<0$, there exists one unstable critical point $\xi_{1}=1 / 2$ of $K+I^{l}$ in $A_{1}^{-1}$.

If $x_{0}>1 / 2$ and $\int_{0}^{1} J^{l}>0$, there exist three critical points $\hat{\xi}_{1}<\xi_{1}=1 / 2,1-\hat{\xi}_{1}$ of $K+I^{l}$ in $A_{1}^{-1}$, such that $\hat{\xi}_{1}$ and $1-\hat{\xi}_{1}$ are stable and $\xi_{1}$ is unstable.

If $x_{0}<1 / 2$ and $\int_{0}^{1} J^{l}>0$, there exists one stable critical point $\xi_{1}=1 / 2$ of $K+I^{l}$ in $A_{1}^{-1}$.

If $x_{0}<1 / 2$ and $\int_{0}^{1} J^{l}<0$, there exist three critical points $\hat{\xi}_{1}<\xi_{1}=1 / 2,1-\hat{\xi}_{1}$ of $K+I^{l}$, such that $\xi_{1}$ is stable and $\hat{\xi}_{1}$ and $1-\hat{\xi}_{1}$ are unstable.

Proof. As in the proof of Theorem 3.2, existence of the above solutions is determined from the equation

$$
\int_{0}^{\xi_{1}} J^{l}(y) d y=\int_{0}^{1-\xi_{1}} J^{l}(y) d y .
$$

Stability and instability then follows from the sign of the Hessian $J^{l}\left(\xi_{1}\right)+J^{l}\left(1-\xi_{1}\right)$. We leave the details to the reader. Note that the $\Gamma$-convergence method implies that for small $\epsilon I_{\epsilon}$ has local minima near stable members of $A_{1}^{-1}$. Whether $I_{\epsilon}$ has, say, mountain-pass solutions near unstable members of $A_{1}^{-1}$ is an open problem. 
We close this section with a discussion of non-existence results. We recall that the Allen-Cahn equation (1.9) with the Neumann boundary condition has no nonconstant stable steady states on $(0,1)$. A natural question is whether $J \geq 0$ (note that $J$ is the entire kernel) and the convexity of $\frac{j u^{2}}{2}+W(u)$ (condition $\mathbf{H 8}$ ) imply that there are no nonconstant local minimizers of $I$.

THEOREM 3.6. Let $J(x, y)=J(x-y)$ and $j(s)+f^{\prime}(s)>0$ for $s \in[-1,1]$. Let $J^{\prime}(x) \leq 0$ for $x \in(0,1)$ and $J(1) \geq 0$. Then any nonconstant solution of $(1.6)$ is unstable, in the sense that it is not a local minimum of $I$.

Proof. With the regularity of $J$ and $f$ we can compute the second variation of I. For every $w, \phi \in L^{2}(0,1)$

$$
\left.\frac{d^{2} I(w+\epsilon \phi)}{d \epsilon^{2}}\right|_{\epsilon=0}=\int_{0}^{1}\left[-J[\phi] \phi+j \phi^{2}+f^{\prime}(w) \phi^{2}\right] d x .
$$

It suffices to show that the right side of (3.9) is $<0$ for $w=u$ and a particular choice of $\phi$. Let us assume that $u$ is a critical point of $I$, i.e., a solution of (1.6). Then (1.6) can be rewritten as

$$
u=(j \cdot+f(\cdot))^{-1}(J[u]) .
$$

The regularity of $J$ and $f$ imply that $u$ is in $C^{1}$. Differentiating (1.6) with respect to $x$, we deduce

$$
-J\left[u^{\prime}\right]+j u^{\prime}+f^{\prime}(u) u^{\prime}=J(x-1)(u(x)-u(1))-J(x)(u(x)-u(0)) .
$$

We now choose $\phi=u^{\prime}$. Multiplying the last equation by $u^{\prime}$ and integrating over $(0,1)$, we obtain

$$
\left.\frac{d^{2} I\left(u+\epsilon u^{\prime}\right)}{d \epsilon^{2}}\right|_{\epsilon=0}=\int_{0}^{1}[J(x-1)(u(x)-u(1))-J(x)(u(x)-u(0))] u^{\prime}(x) d x .
$$

We break up this expression into four integrals and integrate by parts each one of them to get

$$
\begin{gathered}
\int_{0}^{1} J(x-1) u(x) u^{\prime}(x) d x=\frac{1}{2}\left[J(0) u(1)^{2}-J(1) u(0)^{2}\right]-\frac{1}{2} \int_{0}^{1} J^{\prime}(x-1) u(x)^{2} d x, \\
\int_{0}^{1} J(x-1) u(1) u^{\prime}(x) d x=J(0) u(1)^{2}-J(1) u(1) u(0)-\int_{0}^{1} J^{\prime}(x-1) u(1) u(x) d x, \\
\int_{0}^{1} J(x) u(x) u^{\prime}(x) d x=\frac{1}{2}\left[J(1) u(1)^{2}-J(0) u(0)^{2}\right]-\frac{1}{2} \int_{0}^{1} J^{\prime}(x) u(x)^{2} d x, \\
\int_{0}^{1} J(x) u(0) u^{\prime}(x) d x=J(1) u(0) u(1)-J(0) u(0)^{2}-\int_{0}^{1} J^{\prime}(x) u(0) u(x) d x .
\end{gathered}
$$

By completing the squares in the multiples of $J^{\prime}(x)$ and $J^{\prime}(x-1)$ we finally get

$$
\begin{aligned}
\left.\frac{d^{2} I\left(u+\epsilon u^{\prime}\right)}{d \epsilon^{2}}\right|_{\epsilon=0} \equiv R\left(u^{\prime}\right)= & -J(1)(u(1)-u(0))^{2} \\
& -\frac{1}{2} \int_{0}^{1} J^{\prime}(x-1)[u(x)-u(1)]^{2} d x \\
& +\frac{1}{2} \int_{0}^{1} J^{\prime}(x)[u(x)-u(0)]^{2} d x \leq 0 .
\end{aligned}
$$


We now show that actually $R\left(u^{\prime}\right)<0$. Assume that $R\left(u^{\prime}\right)=0$. Then also $R\left(\left|u^{\prime}\right|\right)=$ $0\left(\int_{0}^{1} \int_{0}^{1} J(x-y)\left|u^{\prime}(x) \| u^{\prime}(y)\right| d x d y \geq \int_{0}^{1} \int_{0}^{1} J(x-y) u^{\prime}(x) u^{\prime}(y) d x d y\right)$. Consider the variational problem inf $\|\phi\|_{2}=1, R(\phi)$. If this inf is less than 0 , then there exists some $\phi_{0}$ for which $R\left(\phi_{0}\right)<0$, thus $u$ is unstable. If it is equal to 0 , then since it is achieved at $\phi_{1} \equiv\left|u^{\prime}\right| /\left\|u^{\prime}\right\|_{2}$ we have

$$
-J\left[\left|u^{\prime}\right|\right]+j\left|u^{\prime}\right|+f^{\prime}(u)\left|u^{\prime}\right|=\lambda\left|u^{\prime}\right| .
$$

If $\left|u^{\prime}\right|=0$ at some $x_{0}$, then $-J\left[\left|u^{\prime}\right|\right]\left(x_{0}\right)+j\left(x_{0}\right)\left|u^{\prime}\left(x_{0}\right)\right|=0$, which implies that $\left|u^{\prime}\right| \equiv 0$ (an inductive argument is used if $|\operatorname{suppJ}|<2$ ), a contradiction. So $\left|u^{\prime}\right|>0$. But then it is easily seen that $R\left(u^{\prime}\right)<0$.

The following example shows that condition $J \geq 0$ might be relaxed somewhat.

THEOREM 3.7. If in Theorem 3.6 we set $J(x)=b-m|x|, b, m>0$, and $b \geq 3 m / 4$, then any nonconstant solution of (1.6) is unstable.

Proof. Let $u$ be a solution of (1.6). In (3.10) we break up $-(b-m)(u(1)-u(0))^{2}$ and use $\frac{m}{4}(u(1)-u(0))^{2}$ together with the other two terms in (3.10) to complete the square. We get

$$
\begin{aligned}
\left.\frac{d^{2} I\left(u+\epsilon u^{\prime}\right)}{d \epsilon^{2}}\right|_{\epsilon=0}= & -\left(=b-\frac{3 m}{4}\right)[u(1)-u(0)]^{2} \\
& -m \int_{0}^{1}\left[u(x)-\frac{1}{2}(u(1)+u(0))\right]^{2} d x<0 .
\end{aligned}
$$

Note that $J$ changes sign on $(-1,1)$ if $\frac{3 m}{4} \leq b<m$. $\square$

For some other results in the case $J \geq 0$ we refer the reader to [8].

4. Dynamic solutions in two dimensions. Theorem 2.5 and the existence theorems in Section 3 showed the existence of layered, stable steady solutions of (1.7). Such results should carry over to higher dimensions. However when our approach is used in higher dimensions, layers become hyper-surfaces. The reduced problem of the $\Gamma$-convergence theory, shown in Proposition 2.2, is still an infinitely dimensional problem, which is also sensitive to the shape of the domain $\Omega$. In this section we present some numerical calculation of (1.7) in two dimensions. We solve (1.7) numerically for large time periods. We again assume that $J$ have the form (2.1) with $J^{l}$ invariant, i.e., $J^{l}(x, y)=J^{l}(x-y)$. We only consider the case $J^{l} \geq 0$. We hope to see that some solutions $u(x, t)$ that develop multiple layers as $t \rightarrow \infty$. Then we want to understand how the directions of the layers are affected by $J$ and the domain $\Omega$. Let us first explain the numerical method used here.

We place the domain $\Omega$ inside a square $[0, d] \times[0, d]$. Discretize this square into a $M \times M$ lattice $\{(l, m): l=1,2, \ldots, M, m=1,2, \ldots, M\}$ with $\Delta=d /(M-1)$ being the grid size. Let $D=\{(l, m):((l-1) \Delta,(m-1) \Delta) \in \Omega\}$ be the subset of the lattice points that are inside $\Omega$. Set

$$
u_{l, m}^{n}=u((l-1) \Delta,(m-1) \Delta, n \Delta t),(l, m) \in D .
$$

to be the value of $u\left(x_{1}, x_{2}, t\right)$ at $\left(x_{1}, x_{2}\right)=((l-1) \Delta,(m-1) \Delta)$ and $t=n \Delta t$. Discretize the equation (1.7) to

$$
\frac{u_{l, m}^{n+1}-u_{l, m}^{n}}{\Delta t}=\sum_{(p, q) \in D} J_{l, m, p, q} u_{p, q}^{n} \Delta^{2}-j_{l, m} u_{l, m}^{n}-f\left(u_{l, m}^{n}\right)
$$


for $(l, m) \in D$. This leads to a simple iteration scheme from $u^{n}$ to $u^{n+1}$

$$
u_{l, m}^{n+1}=u_{l, m}^{n}+\Delta t\left[\sum_{(p, q) \in D} J_{l, m, p, q} u_{p, q}^{n} \Delta^{2}-j_{l, m} u_{l, m}^{n}-f\left(u_{l, m}^{n}\right)\right],
$$

Next we address the stability of this scheme. It appears from our numerical experiments that the stability depends mainly on the linear part of the scheme. We use the von Neumann criterion on the linear part of the iteration. Replace $D$ by the entire lattice in plane since the criterion is a local analysis. On the entire plane $j_{l, m}$ becomes a constant independent of $(l, m)$ since $J_{l, m, p, q}=J_{l-p, m-q}$ depends on $l-p$ and $m-q$ only. Let $u_{l, m}^{n}=\xi^{n} \exp \left(i\left(k_{1}, k_{2}\right) \cdot(l, m) \Delta^{2}\right)$ be a solution of

$$
u_{l, m}^{n+1}=u_{l, m}^{n}+\Delta t\left[\sum_{p, q=-\infty}^{\infty} J_{l-p, m-q} u_{p, q}^{n} \Delta^{2}-j u_{l, m}^{n}\right]
$$

Here $\xi^{n} \exp \left(i\left(k_{1}, k_{2}\right) \cdot(l, m) \Delta^{2}\right)$ is thought as a wave of the wave vector $\left(k_{1}, k_{2}\right)$ and magnitude $\xi^{n}$. Insert this expression to (4.4) to obtain

$$
\begin{aligned}
& \xi^{n+1} e^{i\left(k_{1}, k_{2}\right) \cdot(l, m) \Delta^{2}} \\
& =\xi^{n} e^{i\left(k_{1}, k_{2}\right) \cdot(l, m) \Delta^{2}}+\Delta t\left[\sum_{p, q=-\infty}^{\infty} J_{l-p, m-q} \xi^{n} e^{\left(k_{1}, k_{2}\right) \cdot(p, q) \Delta^{2}} \Delta^{2}\right. \\
& \left.\quad-j \xi^{n} e^{i\left(k_{1}, k_{2}\right) \cdot(l, m) \Delta^{2}}\right],
\end{aligned}
$$

which is simplified to

$$
\xi=1-2 \Delta t \sum_{p, q=-\infty}^{\infty} J_{p, q} \sin ^{2}\left(\frac{\left(k_{1}, k_{2}\right) \cdot(p, q) \Delta^{2}}{2}\right) \Delta^{2} .
$$

The von Neumann stability criterion states that a scheme is stable if $|\xi| \leq 1$ for any $\left(k_{1}, k_{2}\right)$. Applying this to (4.5), we obtain

$$
\left|1-2 \Delta t \sum_{p, q=-\infty}^{\infty} J_{p, q} \sin ^{2}\left(\frac{\left(k_{1}, k_{2}\right) \cdot(p, q) \Delta^{2}}{2}\right) \Delta^{2}\right| \leq 1 .
$$

It turns out that (4.6) can be met even for rather large $\Delta t$. For example, when $J_{p, q} \geq 0$,

$$
1-2 \Delta t \sum_{p, q=-\infty}^{\infty} J_{p, q} \Delta^{2} \leq \xi \leq 1 .
$$

So the scheme is stable provided $\Delta t \sum_{p, q=-\infty}^{\infty} J_{p, q} \Delta^{2} \leq 1$. Also note that $\sum_{p, q=-\infty}^{\infty} J_{p, q} \Delta^{2}$ is the discretization of $\int_{\mathbf{R}^{2}} J$, so (4.6) is like

$$
\Delta t \leq \frac{1}{\int_{\mathbf{R}^{2}} J(x, y)} .
$$

Interestingly $\Delta$, the grid size, does not enter (4.7). We will use (4.7) as a guideline to set $\Delta t$, even if $J$ is not everywhere non-negative. 

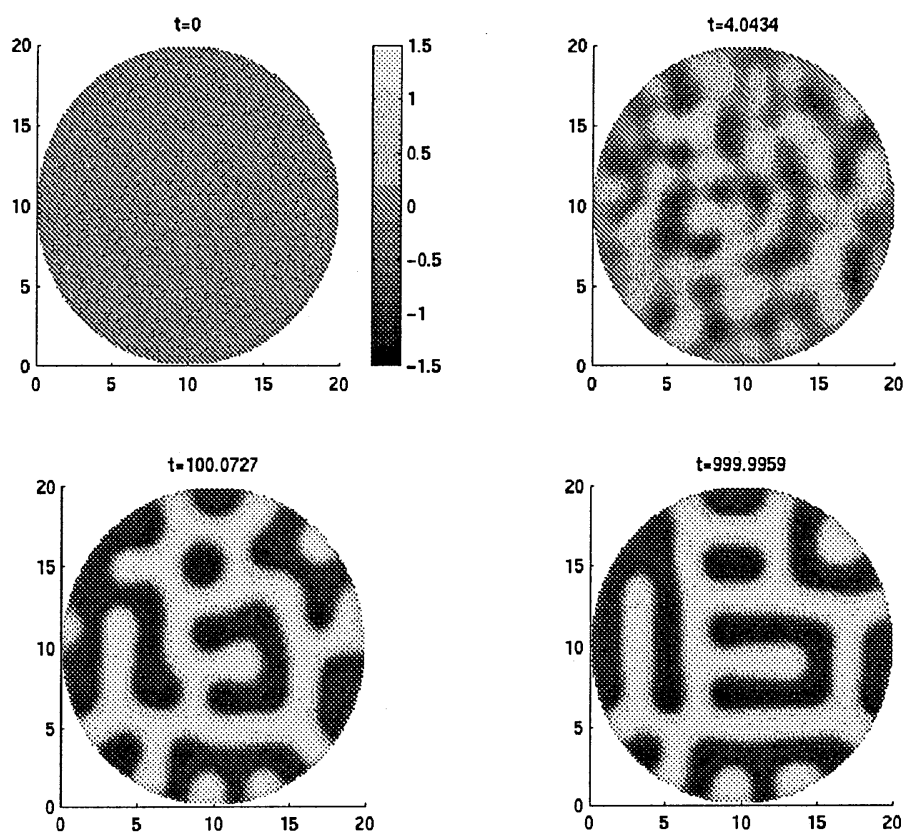

FIG. 4.1. An isotropic $J$ on a disk.

In this paper, $\int_{\mathbf{R}^{2}} J$ is of order 1 , so (4.7) is easily satisfied. The stability of the iteration scheme (4.3) will not be an issue. We will make $\Delta t \sim \Delta$ only to ensure accuracy of the iteration.

As a comparison we point out that the corresponding explicit scheme for the local bistable equation (1.9) requires a much harder to meet von Neumann stability condition $\frac{2 \Delta t}{\Delta^{2}} \leq 1$.

As in the rest of this paper, we have taken the kernel $J(x, y)$ to have the form $J(x-y)=J\left(x_{1}-y_{1}, x_{2}-y_{2}\right)$ for 2 -dimensional vectors $x=\left(x_{1}, x_{2}\right)$ and $y=\left(y_{1}, y_{2}\right)$, so no influence comes from the boundary or the exterior of $\Omega$. Before we present any simulation we note that $J$ can be either isotropic or anisotropic. $J$ is isotropic if $J(x)=J\left(x_{1}, x_{2}\right)$ depends on the magnitude of $\left(x_{1}, x_{2}\right)$ only, and anisotropic if $J\left(x_{1}, x_{2}\right)$ depends on both the magnitude and the direction of $\left(x_{1}, x_{2}\right)$.

The local term in all the simulations is taken to be $W(u)=\frac{1}{4}\left(u^{2}-1\right)^{2}$, so it is a balanced double well function without any $\epsilon$-perturbation, and $f(u)=u^{3}-u$. The size of perturbation is set to be $\epsilon=0.1$. For (4.3) the grid size is $\Delta=0.1$, and $\Delta t=0.1$.

We first consider the isotropic case. The first simulation is on the disk $\Omega=$ $\left\{\left(x_{1}, x_{2}\right): x_{1}^{2}+x_{2}^{2}<10^{2}\right\}$. The kernel is

$$
J\left(x_{1}, x_{2}\right)=\frac{1}{\epsilon^{2}} \exp \left(-\frac{\sqrt{x_{1}^{2}+x_{2}^{2}}}{\epsilon}\right)-\epsilon \exp \left(-\sqrt{0.5 x_{1}^{2}+0.5 x_{2}^{2}}\right) .
$$

It has the form of (2.1) as in Section 2. It changes sign, is positive for $\left(x_{1}, x_{2}\right)$ close to $(0,0)$, and negative otherwise. We take the initial data to be a small perturbation 

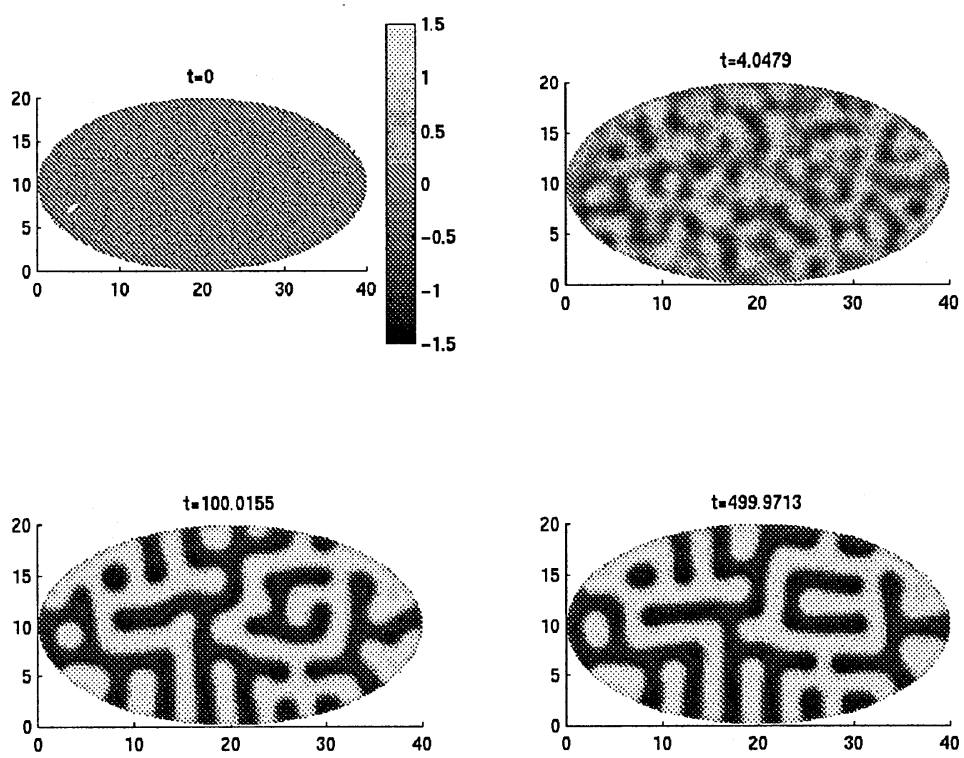

Fig. 4.2. An isotropic $J$ on an ellipse.

of $u=0$. Figure 4.1 is a simulation of $u(x, t)$ at approximately $t=0,4,100,1000$, which shows the formation of a labyrinthine pattern. The colorbar next to the first plot gives the gray scale from -1.5 to 1.5 , which represents the values of $u$ in the four plots. The initial state that $u \approx 0$ is quite unstable, so $u(x, t)$ soon tends to -1 or 1 for most $x$. The exception to such behavior is when $x$ happens to be on the region where $u$ transits between -1 and 1 . Such $x$ 's quickly form a number of curves whose width is of order $\epsilon$. Gradually these curves become more and more straight. Near the boundary the curves tend to be perpendicular to the boundary of $\Omega$. However away from the boundary they do not lie along the same direction. This is because neither $J$ nor the circular domain $\Omega$ prefers any particular overall direction for the layers. The width of a subdomain where $u \approx-1$ is about the same as the width of a subdomain where $u \approx 1$, due to the fact that $W$ is perfectly balanced.

The second simulation explores the influence of the shape of the domain on the direction of the interfaces. We take $\Omega=\left\{\left(x_{1}, x_{2}\right):\left(x_{1} / 20\right)^{2}+\left(x_{2} / 10\right)^{2}<1\right\}$ to be an ellipse, and the same $J$ of (4.8). Figure 4.2 shows a simulation of (1.7) whose initial data is again $u(x, 0) \approx 0$, for 500 time units. This time we also find a labyrinthine pattern. Again the layers tend to be perpendicular to the boundary of $\Omega$ over there. The uneven nature of the boundary of $\Omega$ forces more layers to be in the $x_{2}$ - direction than in the $x_{1}$-direction.

Finally we consider the anisotropic case. This time we take $\Omega=\left\{\left(x_{1}, x_{2}\right)\right.$ : $\left.x_{1}^{2}+x_{2}^{2}<10^{2}\right\}$, so the geometry of $\Omega$ does not prefer any particular direction. Then we take

$$
J\left(x_{1}, x_{2}\right)=\frac{1}{\epsilon^{2}} \exp \left(-\frac{\sqrt{x_{1}^{2}+x_{2}^{2}}}{\epsilon}\right)-\epsilon \exp \left(-\sqrt{x_{1}^{2}+0.5 x_{2}^{2}}\right)
$$



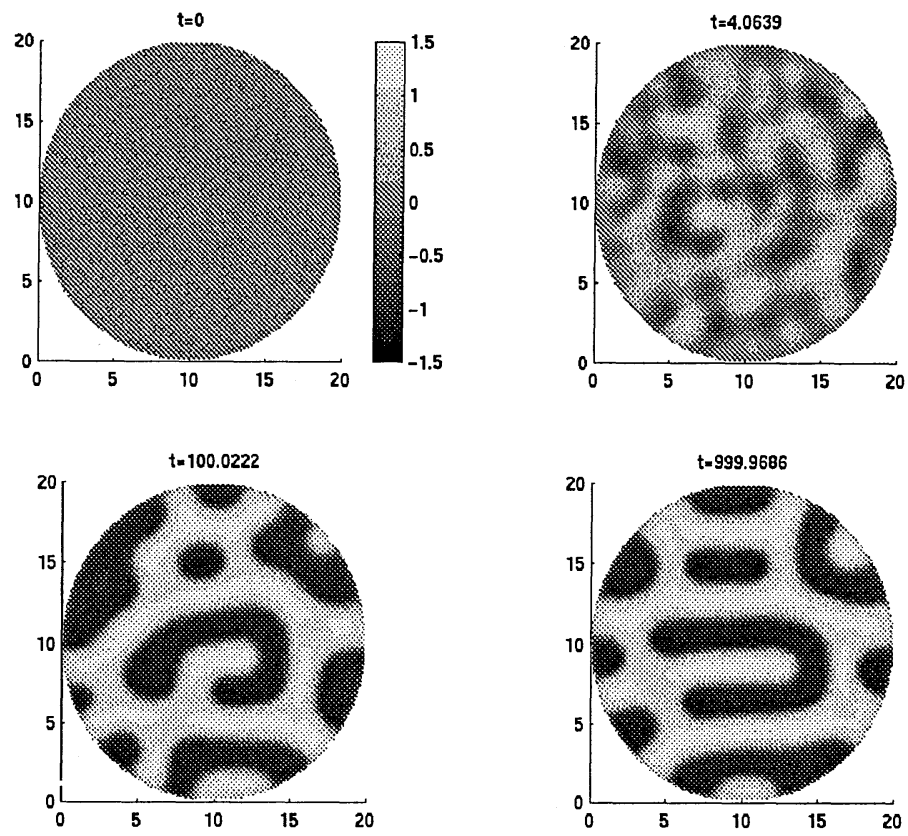

FIG. 4.3. An anisotropic $J$ on a disk.

with the anisotropy built in the second term. We again take the initial data to be $u(x, 0) \approx 0$ and compute for 1000 time units. As shown in Figure 4.3 after the labyrinthine pattern is stabilized, the interfaces in $x_{1}$ direction tend to be longer than the interfaces in $x_{2}$ direction. In other words the anisotropic $J$ in (4.9) prefers oscillation in $x_{2}$-direction more than in $x_{1}$ direction. This is consistent with the fact that in (4.9)

$$
J^{l}\left(x_{1}, x_{2}\right)=\exp \left(-\sqrt{x_{1}^{2}+0.5 x_{2}^{2}}\right)
$$

so $J^{l}$ decreases slower in $x_{2}$ direction than in $x_{1}$ direction, and $J^{l}$ is largely responsible for the oscillatory behavior of the solution, in which $x_{2}$ direction is more dominant.

\section{REFERENCES}

[1] G. Alberti AND G. BelletTini, A non-local anisotropic model for phase transitions: asymptotic behavior of rescaled energies, Euro. J. Appl. Math., 9 (1998), pp. 261-284.

[2] S. Allen AND J. W. CAHN, A microscopic theory for antiphase boundary motion and its application to antiphase domain coarsening, Acta Metall., 27 (1979), pp. 1084-1095.

[3] P. W. BAtes, P. C. FIfe, X. Ren, AND X. WANG, Traveling waves in a convolution model for phase transitions, Arch. Rational. Mech. Anal., 138 (1997), pp. 105-136.

[4] P. W. BATES AND A. ChMAJ, An integrodifferential model for phase transitions: stationary solutions in higher space dimensions, J. Statist. Phys., 95 (1999), pp. 1119-1139.

[5] P. W. Bates AND A. ChMAJ, On a discrete convolution model for phase transitions, Arch. Rational. Mech. Anal., 150 (1999), pp. 281-305.

[6] A. ChMAJ AND X. REN, Homoclinic solutions of an integral equation: existence and stability, J. Differential Equations, 155 (1999), pp. 17-43. 
[7] A. ChmAJ AND X. ReN, Multiple layered solutions of the nonlocal bistable equation, Physica D, 147 (2000), pp. 135-154.

[8] A. CHMAJ AND X. ReN, The nonlocal bistable equation: stationary solutions on a bounded interval, Electronic J. Differential Equations, 2002 (2002), pp. 1-12.

[9] E. De GIoRgi, Sulla convergenza di alcune successioni di integrali del tipo della'area, Rendiconti di Matematica, 8 (1975), pp. 277-294.

[10] L. C. Evans AND R. F. GARIEPY, Measure theory and fine properties of functions, CRC Press, Boca Raton New York London Tokyo, 1992.

[11] X. REN AND L. TRuskinovsky, Finite scale microstructures in nonlocal elasticity, J. Elasticity, 59 (2000), pp. 319-355.

[12] X. REN AND J. WEI, On the multiplicity of solutions of two nonlocal variational problems, SIAM J. Math. Anal., 31 (2000), pp. 909-924.

[13] R. KoHN AND P. Sternberg, Local minimizers and singular perturbations, Proc. Roy. Soc. Edinburgh Sect., A 111 (1989), pp. 69-84.

[14] J. D. MurRAY, Mathematical Biology, Springer-Verlag, 1993. 
A. J. J. CHMAJ AND X. REN 